\title{
Insights about the structure of farnesyl diphosphate synthase (FPPS) and the activity of bisphosphonates on the proliferation and ultrastructure of Leishmania and Giardia
}

Ana Paula R. Gadelha 1,2,3, Claudia Maia Brigagao ${ }^{3}$, Martha Barros da Silva $^{3}$, Aline Beatriz Mello Rodrigues ${ }^{4}$, Ana Carolina Ramos Guimarães ${ }^{4}$, Fernando Paiva ${ }^{5}$, Wanderley de Souza ${ }^{3}$ and Cristina Henriques ${ }^{3,6,7^{*}}$ (D)

\begin{abstract}
Background: The enzyme farnesyl diphosphate synthase (FPPS) is positioned in the intersection of different sterol biosynthesis pathways such as those producing isoprenoids, dolichols and ergosterol. FPPS is ubiquitous in eukaryotes and is inhibited by nitrogen-containing bisphosphonates (N-BP). N-BP activity and the mechanisms of cell death as well as damage to the ultrastructure due to N-BP has not yet been investigated in Leishmania infantum and Giardia. Thus, we evaluated the effect of N-BP on cell viability and ultrastructure and then performed structural modelling and phylogenetic analysis on the FPPS enzymes of Leishmania and Giardia.
\end{abstract}

Methods: We performed multiple sequence alignment with MAFFT, phylogenetic analysis with MEGA7, and 3D structural modelling for FPPS with Modeller 9.18 and on I-Tasser server. We performed concentration curves with N-BP in Leishmania promastigotes and Giardia trophozoites to estimate the $\mathrm{IC}_{50}$ via the MTS/PMS viability method. The ultrastructure was evaluated by transmission electron microscopy, and the mechanism of cell death by flow cytometry.

Results: The nitrogen-containing bisphosphonate risedronate had stronger anti-proliferative activity in Leishmania compared to other N-BPs with an $\mathrm{IC}_{50}$ of $13.8 \mu \mathrm{M}$, followed by ibandronate and alendronate with $\mathrm{IC}_{50}$ values of $85.1 \mu \mathrm{M}$ and $112.2 \mu \mathrm{M}$, respectively. The effect of N-BPs was much lower on trophozoites of Giardia than Leishmania ( $\mathrm{IC}_{50}$ of $311 \mu \mathrm{M}$ for risedronate). Giardia treated with N-BP displayed concentric membranes around the nucleus and nuclear pyknosis. Leishmania had mitochondrial swelling, myelin figures, double membranes, and plasma membrane blebbing. The same population labelled with annexin- $V$ and 7-AAD had a loss of membrane potential (TMRE), indicative of apoptosis. Multiple sequence alignments and structural alignments of FPPS proteins showed that Giardia and Leishmania FPPS display low amino acid identity but possess the conserved aspartate-rich motifs.

Conclusions: Giardia and Leishmania FPPS enzymes are phylogenetically distant but display conserved protein signatures. The N-BPs effect on FPPS was more pronounced in Leishmania than Giardia. This might be due to general differences in metabolism and differences in the FPPS catalytic site.

Keywords: Bisphosphonates, Farnesyl diphosphate synthase, FPPS, Leishmania, Giardia, Protozoan, Isoprenoid, Isoprenylation, Sterol, Ergosterol

\footnotetext{
*Correspondence: cristina.henriques@fiocruz.br

${ }^{7}$ Laboratório de Biotecnologia e Fisiologia de Infecções Virais-LABIFIV,

Instituto Oswaldo Cruz (IOC, Fiocruz), Av Brasil 4365, Manguinhos, Rio de Janeiro, RJ, Brazil

Full list of author information is available at the end of the article
}

(c) The Author(s) 2020. This article is licensed under a Creative Commons Attribution 4.0 International License, which permits use, sharing, adaptation, distribution and reproduction in any medium or format, as long as you give appropriate credit to the original author(s) and the source, provide a link to the Creative Commons licence, and indicate if changes were made. The images or other third party material in this article are included in the article's Creative Commons licence, unless indicated otherwise in a credit line to the material. If material is not included in the article's Creative Commons licence and your intended use is not permitted by statutory regulation or exceeds the permitted use, you will need to obtain permission directly from the copyright holder. To view a copy of this licence, visit http://creativeco mmons.org/licenses/by/4.0/. The Creative Commons Public Domain Dedication waiver (http://creativecommons.org/publicdomain/ zero/1.0/) applies to the data made available in this article, unless otherwise stated in a credit line to the data. 


\section{Background}

Farnesyl diphosphate synthase (FPPS) is a key enzyme in sterol metabolism. It is positioned at the intersection of different pathways, including those involved in the biosynthesis of isoprenoids, dolichols, ubiquinones and ergosterol/cholesterol. Giardia and other early diverging eukaryotes do not synthesize ergosterol or cholesterol de novo in contrast to Leishmania and trypanosomatids that synthesize ergosterol instead of cholesterol, which is produced by humans and other mammals.

The pathway for ergosterol biosynthesis includes enzymes that differ from cholesterol biosynthesis, making the ergosterol biosynthesis pathway a potential target for chemotherapy [1, 2]. Other pathways and enzymes of sterol metabolism include isoprenoid/prenylation and the dolichol biosynthesis. These pathways are ubiquitous in eukaryotes but have not received much attention. Genomic analysis has facilitated prediction of several metabolic pathways among eukaryotic organisms [3] and these predicted pathways enable comparisons to be made between sterol metabolism in early branching protozoans such as Giardia and Leishmania.

Leishmaniasis is a complex of diseases. There are more than 20 Leishmania species that cause different diseases, i.e. visceral leishmaniasis (VL), cutaneous leishmaniasis (CL) and mucocutaneous leishmaniasis (MCL). Leishmaniasis occurs in 102 countries, and CL is the most common and widespread [4]. More than $70 \%$ of the CL cases occur in 10 countries: Afghanistan, Algeria, Brazil, Colombia, Costa Rica, Ethiopia, the Islamic Republic of Iran, Peru, Sudan and the Syrian Arab Republic [4]. Around $90 \%$ of the global VL cases are reported in only six countries: Bangladesh, Brazil, Ethiopia, India, South Sudan and Sudan. In the Americas, Leishmania infantum is the etiological agent of VL [5], which is lethal if not treated. Brazil has a high burden of CL and VL with an incidence rate of 1.46 and 0.41 cases per 10,000 inhabitants, respectively $[4,6], C L$ cases are widespread throughout the Brazilian national territory and VL cases are reported in 21 states [7]. Leishmaniasis has been spread to previously non-endemic areas including urban centers. Indeed, nearly 1600 Brazilian cities have autochthonous transmission [7].

Giardia is the causative agent of giardiasis. It is a major cause of diarrhea in humans and an important public health problem [8, 9]. Giardia duodenalis (syn. G. intestinalis and G. lamblia) is divided into eight genetic assemblages (A-H) $[10,11]$ and possesses two morphological forms: trophozoites that infect the duodenum; and cysts that facilitate disease transmission by contaminating soil, food, and water following excretion in the feces. Giardia duodenalis assemblages $\mathrm{A}$ and $\mathrm{B}$ are responsible for human giardiasis and these types are globally distributed $[9,10,12]$.
Giardia sterol metabolism is restricted to a few metabolic pathways [13] including the isoprenoid, the dolichol, and the ubiquinone or coenzyme Q (CoQ) pathways. CoQ is a component of the electron transport chain in aerobic organisms such as Leishmania, but is detected at much lower levels in Giardia, which has a poorly developed endomembrane system and lacks organelles including the Golgi and mitochondria [14, 15].

In contrast to Giardia, Leishmania has a complex lifecycle and sterol metabolism. It has adapted to a life-cycle that alternates between the promastigote (the infective form found inside the phlebotomine vector) and the amastigote form that resides inside the macrophages of the mammalian host. Leishmania has a sophisticated endo-membrane system, evolved mitochondria, and possesses the main enzymes and pathways of sterol metabolism. The enzyme profile of sterol metabolism and the presence of sterol-metabolizing gene sequences in the genome of Giardia and Leishmania suggest that the five carbon isoprene units, isopentenyl diphosphate (IPP) and its isomer dimethylallyl diphosphate (DMAPP), are synthesized via the mevalonate pathway (MEV) [3]. The IPP and DMAPP metabolites are substrates of farnesyl diphosphate synthase (FPPS) and lead to production of 15 carbon farnesyl diphosphate (FPP).

FPP is a key intermediate of sterol metabolism with a role in the post-translational modification of proteins via farnesyl transferase as well as in protein prenylation of the Ras superfamily of small GTP-binding proteins. FPP is also the precursor of several biomolecules with distinct biological function including the polyisoprenoids composed of 11 to 23 isoprene units known as dolichols [16].

Dolichols are carriers of N-glycan and glycosylphosphatidylinositol (GPI). They are inserted in the internal membrane of the endoplasmic reticulum (ER) and have a role in post-translational modification of proteins. Leishmania and Giardia produce dolichols with 11 to 12 isoprene units $[17,18]$.

Giardia lost the capacity to synthesize ergosterol and cholesterol de novo during evolution, but it does possess the enzymes of the MEV pathway including FPPS. Comparative analyses based on profiling of sterol biosynthethic enzymes of 46 eukaryotic proteomes showed that farnesyl/geranyl diphosphate synthase (FPPS and GPPS) and farnesyl transferase complex are ubiquitous in all organisms studied, including Giardia. This indicates that isoprenoid production is indispensable for all eukaryotes [3]. Giardia FPPS displays the conserved motifs and protein signatures found in FPPS of other organisms but has low identity with FPPS of humans, and Leishmania as evaluated previously by multiple sequence alignment and phylogenetic analyses [19]. In L. major, the FPPS structure was elucidated via crystallography [20]. 
Functional characterization of the recombinant FPPS has demonstrated that the enzyme is strongly inhibited by nitrogen-containing bisphosphonates (N-BP) such as risedronate [21, 22]. N-BPs have been the frontline treatment for bone disorders including osteoporosis, tumorassociated bone disease, and Paget's disease [23]. N-BPs lead to depletion of FPP and GGPP isoprenoids and required prenylation of small GTPase proteins. The failure of protein prenylation due to N-BP is one of the main mechanisms behind decreased bone resorption by osteoclasts [24]. Bisphosphonates are also shown to be active against some protozoans [19, 25] but have not been tested on L. infantum and G. duodenalis. Furthermore, the mechanism of death and the effect on mitochondrial function and ultrastructure due to N-BP treatment has not been rigorously explored in parasitic protozoans.

We performed molecular modelling of FPPS sequences from $L$. infantum and from the distantly related FPPS enzyme of Giardia. Phylogenetic analysis of Leishmania FPPS and of different isolates of Giardia was also performed. We tested the effect of N-BPs on the protozoan L. infantum and Giardia to evaluate its effects on protozoan proliferation, viability and ultrastructure. Our results suggest that the isoprenoid pathway may represent an interesting target for evaluating mechanisms of cell death and a target for anti-parasitic drugs.

\section{Methods}

\section{Parasite culture}

Promastigotes of Leishmania infantum MHOM/BR/74/ PP75 (IOCL0579, CLIOC-http://clioc.fiocruz.br) were grown in Schneider's medium (Sigma-Aldrich, São Paulo, Brazil) supplemented with $10 \%$ fetal calf serum (FCS) maintained at $26{ }^{\circ} \mathrm{C}$ until the logarithmic growth stage. Trophozoites of G. duodenalis WB strain (clone C6; ATCC No. 30957) were cultivated in TYI-S-33 medium [26], pH 7.2, supplemented with $0.1 \%$ bovine bile and $10 \%$ FCS. Cultures were maintained at $37^{\circ} \mathrm{C}$.

\section{Multiple sequence alignment and phylogenetic reconstruction}

A Basic Local Alignment Search Tool (BLASTp) search was performed with FPPS sequences of proteins experimentally characterized and deposited in the Protein Data Bank (PDB) and UniProtKB/Swiss-Prot (Table 1).

The sequences were download in FASTA format to perform multi-alignment and phylogenetic analysis. Multiple sequence alignment was performed using MAFFT v7 (EMBL-EBI search and sequence analysis tool; https://www.ebi.ac.uk/Tools/msa/mafft/) applying the BLOSUM62 matrix 1.53 gap open penalty and default parameter settings [27]. Phylogenetic analysis of FPPS sequences was performed in MEGA 7 [28, 29]. The evolutionary history was inferred by the Maximum Likelihood method based on the JTT matrix-based model [30]. The bootstrap consensus tree inferred from 1000 replicates was used to represent the evolutionary history of the analyzed taxa [31]. Initial tree(s) for the heuristic search were obtained automatically by applying Neighbor-Join and BioNJ algorithms to a matrix of pairwise distances estimated using a JTT model. The topology with superior log likelihood values were then selected.

\section{Theoretical modeling of FPPS}

The amino acid sequences of Leishmania infantum and Giardia intestinalis (Assemblage A isolate WB; gene: GL50803_6633) related to farnesyl pyrophosphate synthase were used to construct the 3D theoretical structure models of this enzyme. These sequences were subjected to BLASTp searches (https://blast.ncbi.nlm.nih.gov/Blast .cgi?PAGE = Proteins) to identify potential template structures from PDB for prediction of Giardia and Leishmania FPPS structures. Regarding the FPPS target sequence for L. infantum, 50 models were generated with the standard auto model routine and optimized via the variable target function method (VTFM) until 300 iterations were achieved using Modeller version 9.18 [32] and PDB 4JZB [20] as the template. The model with the lowest discrete optimized protein energy (DOPE) value was selected.

The system's energy was minimized using Chimera software by applying the default parameters (http://www. rbvi.ucsf.edu/chimera). Additionally, the sequence of Giardia WB FPPS was submitted to the I-Tasser server (https://zhanglab.ccmb.med.umich.edu/I-TASSER/) to obtain a model via a threading prediction. Both models were submitted to the SAVES server (http://servi cesn.mbi.ucla.edu/SAVES/) to be evaluated by validation programs (Ramachandran plot, ERRAT and Verify3D). The three-dimensional structures were generated using PyMOL [33]. All structures have electrostatic surface maps created using PyMOL with the APBS plugin (default parameters) predicted by assuming a pH 7.0.

\section{In vitro assays of bisphosphonate in Leishmania and Giardia}

The N-BP and inhibitors of FPPS (Sigma-Aldrich) including alendronate sodium trihydrate (A4978), ibandronate sodium salt (I5784), neridronate (N6037), pamidronate disodium salt hydrate (P2371), and risedronate sodium (SML0650) were diluted in water to prepare stock solutions. Concentration curves were performed on Leishmania promastigotes at $10^{6}$ promastigotes $/ \mathrm{ml}$ in Schneider's medium supplemented with 10\% FCS. Concentration curves were performed for each N-BP with increasing concentrations: 10, 20, 40, 80, 100, 200 and $400 \mu \mathrm{M}$. 
Table 1 FPPS protein sequences

\begin{tabular}{|c|c|c|}
\hline Organism & Accession number & References \\
\hline Homo sapiens & P14324.4; 4 LFV & [39] \\
\hline Saccharomyces cerevisiae & P08524. $2^{\mathrm{a}}$ & {$[50]$} \\
\hline Leishmania major & Q4QBL1 $^{\mathrm{a}} ; 4 J Z X^{\mathrm{b}} ; 4 J Z \mathrm{~B}^{\mathrm{b}}, 4 \mathrm{~K} 10^{\mathrm{b}} ;$ XP_001683287.1 ${ }^{\mathrm{c}}$ & {$[20]$} \\
\hline Trypanosoma cruzi & EKG07068.1 $1^{\mathrm{e}} ; 1 \mathrm{YHK}^{\mathrm{b}} ; 1 \mathrm{YHL}^{\mathrm{b}} ; 1 \mathrm{YHM}^{\mathrm{b}}$ & {$[51]$} \\
\hline Trypanosoma brucei brucei TREU927 & $\mathrm{Q}^{2} 7 W F 1^{\mathrm{a}} ; 2 \mathrm{EWG} \mathrm{G}^{\mathrm{b}}$ XP_845959.1 ${ }^{\mathrm{c}}$ & {$[52]$} \\
\hline Escherichia coli (strain K12) & $\mathrm{P} 22939^{\mathrm{a}} ;\left.1 \mathrm{RQ}\right|^{\mathrm{b}}$ & [53] \\
\hline Arabidopsis thaliana (thale cress) & Q43315; NP_193452.1 ${ }^{\mathrm{c}}$ & {$[54]$} \\
\hline Leishmania donovani & $A B \mid 16061.1^{e}$ & \\
\hline Leishmania infantum JPCM5 & E9AH04 ${ }^{a} ;$ XP_003392505. $1^{c}$ & \\
\hline Leishmania mexicana & XP_003875590.1 ${ }^{\mathrm{c}}$ & \\
\hline Leishmania braziliensis MHOM/BR/75/M2904 & $\mathrm{A} 4 \mathrm{HCH} 8^{\mathrm{a}} ; \mathrm{XP}_{-} 001565042.1^{\mathrm{c}}$ & \\
\hline Crithidia fasciculata strain Cf-Cl & CFAC1_240021200.1-p1 ${ }^{d}$ & \\
\hline Giardia intestinalis ATCC 50803 & XP_001709477.1'; GL50803_6633-t26_1-p1 ${ }^{\mathrm{f}}$ & \\
\hline Giardia intestinalis ATCC 50581 & EES99536.1 ${ }^{\mathrm{e}} ; \mathrm{GL} 50581$ 3281-t26_1-p1 ${ }^{\mathrm{f}}$ & \\
\hline Giardia lamblia P15 & EFO63579.1 ${ }^{\mathrm{e}}$;GLP15_4726-t26_1-p1 ${ }^{\mathrm{f}}$ & \\
\hline Shigella & WP_000347239.1 ${ }^{c}$ & \\
\hline Enterobacteriaceae & WP_000347220.1 ${ }^{c}$ & \\
\hline
\end{tabular}

a UniProtKB/Swiss-Prot database

b PDB database

c NCBI database

d TriTrypDB database

e GenBank database

f GiardiaDB database

Promastigotes were incubated with each compound for $72 \mathrm{~h}$ at $26^{\circ} \mathrm{C}$.

Giardia duodenalis trophozoites were cultivated in Eppendorf tubes containing $10^{5}$ trophozoites $/ \mathrm{ml}$ in TYIS-33 medium supplemented with $10 \%$ FCS. Concentration curves were performed for each N-BP inhibitor with increasing concentrations: 10, 50, 100, 200, 500 and $1000 \mu \mathrm{M}$ for $48 \mathrm{~h}$ at $37^{\circ} \mathrm{C}$.

The $\mathrm{IC}_{50}$ was estimated using the viability method using tetrazolium 3-(4,5-dimethyl-2-thiazolyl)-5-(3carboxymethoxyphenyl)-2-(4-sulfophenyl)-2H (MTS) and 5-methyl-phenazinium methyl sulfate (PMS) [34]. Thereafter, the $\mathrm{IC}_{50}$ was estimated by non-linear regression using the Sigma plot software.

\section{Tetrazolium salt-based viability assay}

Parasite cultures of Leishmania promastigotes and G. duodenalis trophozoites were centrifuged at $2000 \times g$ for $10 \mathrm{~min}$ at $4{ }^{\circ} \mathrm{C}$. Before centrifugation, G. duodenalis trophozoites were placed on ice for $10 \mathrm{~min}$ and then shaken to detach the parasites. After centrifugation, the medium was removed and the pellet was suspended with the same volume of saline buffer composed of $21 \mathrm{mM}$ HEPES, $0.7 \mathrm{mM} \mathrm{Na}_{2} \mathrm{PO}_{4}, 137 \mathrm{mM} \mathrm{NaCl}, 5 \mathrm{mM} \mathrm{KCl}$ at
$\mathrm{pH} 7.4$ supplemented with $6 \mathrm{mM}$ glucose (SBG). For each condition, $100 \mu \mathrm{l}$ of each parasite cell suspension was transferred in triplicate to a 96-well plate. To perform the negative control, $100 \mu \mathrm{l}$ of each parasite suspension was transferred in duplicate and fixed with $0.4 \%$ paraformaldehyde. Afterwards, $20 \mu \mathrm{l}$ of MTS/PMS mixture was added to each well containing $100 \mu \mathrm{l}$ of protozoan in buffer SBG [34]. To produce the MTS/PMS mixture, $50 \mu \mathrm{l}$ PMS (Sigma-Aldrich; P9625) stock solution was added to $1 \mathrm{ml}$ of MTS stock solution (CellTiter 96 AQueous MTS Reagent Powder, G1112; Promega, São Paulo, Brazil).

\section{Electron microscopy}

To evaluate the ultrastructure of protozoans treated with NB-Ps, Leishmania promastigotes and G. duodenalis trophozoites were incubated and fixed with $2.5 \%$ glutaraldehyde, $2.0 \%$ paraformaldehyde in cacodylate buffer, and post-fixed with $1 \%$ osmium tetroxide and $0.8 \%$ potassium ferrocyanide in $0.1 \mathrm{M}$ cacodylate buffer $(\mathrm{pH} 7.4)$ for $1 \mathrm{~h}$ at room temperature. The samples were then washed, dehydrated in acetone, and embedded in Epon. Thin sections were stained with uranyl acetate and lead citrate and observed via transmission electron microscopy (Tecnai ${ }^{\mathrm{TM}}$ Spirit TEM; FEI Company, São Paulo, Brazil). 


\section{Flow cytometry analysis of $L$. infantum promastigotes} treated with alendronate

Programmed cell death was evaluated in L. infantum promastigotes treated for 24,48 and $72 \mathrm{~h}$ with $100 \mu \mathrm{M}$ alendronate and $10 \mu \mathrm{M}$ miltefosine (M5571; Merck, São Paulo, Brazil) as a control. After treatment, parasites were analyzed by flow cytometry (BD Accuri ${ }^{\mathrm{TM}}$ C6; BD Biosciences, São Paulo, Brazil), and the BD Accuri C6 Software. Early and late apoptotic processes were distinguished using the vital dye 7-amino-actinomycin (7-AAD; BD Pharmingen, São Paulo, Brazil) as well as Annexin-V-FITC that binds to the exposed phospholipid phosphatidylserine (PS) in membranes (FITC Annexin V Apoptosis Detection Kit I; BD Pharmingen). Briefly, 1 $\mathrm{ml}$ of $L$. infantum culture with approximately $2-8 \times 10^{6} \%$ $\mathrm{ml}$ promastigotes was centrifuged at $2000 \times g$ for $10 \mathrm{~min}$, and the pellet was suspended in $100 \mu \mathrm{l}$ of binding buffer according to the manufacturer's suggestions. The $5 \mu \mathrm{l}$ of annexin-V-FITC and/or $5 \mu \mathrm{l}$ of 7-AAD $(10 \mathrm{mg} / \mathrm{ml})$ was added and incubated for $15 \mathrm{~min}$. We then added $400 \mu \mathrm{l}$ of binding buffer to a final volume of $500 \mu \mathrm{l}$. The samples were analyzed with a flow cytometer (BD Accuri C6, BD Biosciences), and 20,000 events were acquired. Controls were performed in promatigotes whose membranes had been permeabilized with $0.5 \%$ Triton X-100 for $15 \mathrm{~min}$ followed by incubation with 7-AAD (FL3) and/ or Annexin-V-FITC (FL1). The forward and side scatter plots $(\mathrm{FSC}-\mathrm{H} \times \mathrm{SSC}-\mathrm{H})$ were used to evaluate the promastigote population with respect to cellular volume and shape.

The L. infantum promastigotes were incubated with $100 \mathrm{nM}$ tetramethylrhodamine ethyl ester (TMRE, BD Pharmingen) to investigate the mitochondrial membrane potential after treatment with bisphosphonates. A stock solution of TMRE (1 mM) was prepared in DMSO and stored at $-20^{\circ} \mathrm{C}$. Thereafter, $1 \mathrm{ml}$ of promastigotes incubated with bisphosphonates or miltefosine for 48 or $72 \mathrm{~h}$, approximately $5-8 \times 10^{6} / \mathrm{ml}$, were centrifuged at $2000 \times g$ for $10 \mathrm{~min}$. The pellets were suspended in $1 \mathrm{ml}$ of saline buffer $\left(137 \mathrm{mM} \mathrm{NaCl}, 5 \mathrm{mM} \mathrm{KCl}, 0.7 \mathrm{mM} \mathrm{Na} \mathrm{HPO}_{4}\right.$, $6 \mathrm{mM}$ glucose, and $21 \mathrm{mM}$ HEPES, $\mathrm{pH} 7.3)$ and $0.1 \mu \mathrm{l}$ of TMRE stock solution (FL2) was added and incubated for $15 \mathrm{~min}$ at room temperature. The promastigotes were then centrifuged, washed, and evaluated by flow cytometry (BD Accuri C6, BD Biosciences).

\section{Results}

\section{Giardia and Leishmania FPPS sequence analysis}

Giardia WB FPPS protein sequences display low identity to Homo sapiens (27.8\%), Saccharomyces cerevisiae (29.9\%), L. infantum (27.7\%), L. major (28\%) and Escherichia coli (20.9\%). FPPS sequences of L. infantum and $L$. major had a higher identity ( 88\%) to Crithidia fasciculate, than to Trypanosoma cruzi and T. brucei ( 63\%). The identity to higher-order eukaryotes was also lower; 43\% for Arabidopsis thaliana, 37\% for Homo sapiens and $34 \%$ for $S$. cerevisiae, with a coverage of $70-80 \%$. The FPPS protein sequences of Giardia Assemblage A isolate WB displayed 97\% identity to Giardia Assemblage B isolate GS, and 83\% identity to Giardia Assemblage E isolate P15.

Despite the low identity of Giardia FPPS with Leishmania and other eukaryotes, Giardia FPPS has conserved aspartate-rich motifs that are characteristic of FPPS enzymes. The first aspartate (D)-rich motif (FARM) is composed of DDXXD and is found in eukaryotic organisms (Fig. 1a, b). The second aspartate rich motif (SARM) has the DDXXD sequence (Fig. 1c, d). Crystallographic studies have shown that these motifs face each other and create a binding pocket. Both motifs are also involved in the catalytic site of the FPPS enzyme of several organisms, even in E. coli.

The FARM sequence motif is conserved from Homo sapiens to bacteria (Fig. 1a) and display two conserved arginine (R) residues and one lysine (K) downstream. These sequences are involved in binding of the substrate and coordination with $\mathrm{Mg}^{+2}$ ions (Fig. 1a). A comparison of the L. major, L. infantum, and Giardia WB FARM motif indicates that the conserved aspartate residues (bold) are $\operatorname{Asp}^{98}(\mathrm{D}), \operatorname{Asp}^{99}(\mathrm{D}), \operatorname{Ile}^{100}(\mathrm{I}), \operatorname{Met}^{101}(\mathrm{M})$ and $\mathbf{A s p}^{102}(\mathrm{D})$ in Leishmania, and $\mathbf{A s p}^{120}(\mathrm{D}), \mathbf{A s p}^{121}(\mathrm{D})$, $\mathrm{Val}^{122}(\mathrm{~V})$, Ile ${ }^{123}(\mathrm{I})$ and $\mathbf{A s p}^{124}(\mathrm{D})$ in Giardia WB. Other conserved residues are two arginine units (bold): $\operatorname{Arg}^{107}(\mathrm{R})$ and $\mathbf{A r g}^{108}(\mathrm{R})$ in Leishmania and $\mathbf{A r g}^{129}(\mathrm{R})$ and $\operatorname{Arg}^{130}(\mathrm{R})$ in Giardia WB (Fig. 1b) as corroborated by InterPro (https://www.ebi.ac.uk/interpro/).

A comparison of the fifth and fourth amino acid residues before the FARM motif involved a chain length determination of the enzymatic product (GPP, FPP or GGPP). These are composed of $\mathrm{His}^{93}(\mathrm{H}), \mathrm{Phe}^{94}(\mathrm{~F})$, $\mathrm{Leu}^{95}(\mathrm{~L}), \mathrm{Val}^{96}(\mathrm{~V})$ and $\mathrm{Glu}^{97}(\mathrm{E})$ in Leishmania as well as $\operatorname{Ala}^{115}(\mathrm{~A}), \operatorname{Phe}^{116}(\mathrm{~F}), \operatorname{Leu}^{117}(\mathrm{~L}), \operatorname{Met}^{118}(\mathrm{M})$ and $\operatorname{Ile}^{119}(\mathrm{I})$ in Giardia (Fig. 1b). Phenylalanine (F) is a key residue involved in limiting the product chain length in Leishmania. The tyrosine $(\mathrm{Y})$ residue has the same role in trypanosomes (Fig. 1b).

Multiple sequence alignment was performed to compare the SARM motif, which is composed of conserved aspartate residues in different organisms (Fig. 1c) and to compare the SARM motif and surrounding residues from trypanosomatids and Giardia (Fig. 1d). A sequence comparison between Leishmania and Giardia WB demonstrates the following conserved residues (bold) in Leishmania; $\operatorname{Gln}^{167}(\mathrm{Q}), \mathbf{A s p}^{170}(\mathrm{D}), \mathbf{L y s}^{207}(\mathrm{~K})$, $\operatorname{Thr}^{208}(\mathrm{~T}), \operatorname{Tyr}^{211}(\mathrm{Y}), \mathbf{A s p}^{250}(\mathrm{D}), \mathbf{A s p}^{251}(\mathrm{D}), \mathbf{A s p}^{254}(\mathrm{D})$ 
and $\operatorname{Asp}^{268}(D)$. Similar residues were also found in Giardia such as $\operatorname{Gln}^{187}(\mathrm{Q}), \quad \mathbf{A s p}^{190}(\mathrm{D}), \quad \mathbf{L y s}^{212}(\mathrm{~K})$, $\operatorname{Thr}^{213}(\mathrm{~T}), \operatorname{Tyr}^{216}(\mathrm{Y}), \mathbf{A s p}^{285} \mathrm{D}, \mathbf{A s p}^{286} \mathrm{D} \mathbf{A s p}^{289}(\mathrm{D})$ and $\operatorname{Asp}^{302}(\mathrm{D})$. The lysine (K) residues downstream of the SARM motif were $\mathbf{L y s}^{264}(\mathrm{~K})$ and $\mathbf{L y s}^{273}(\mathrm{~K})$ in Leishmania and $\mathbf{L y s}^{298}(\mathrm{~K})$ and $\mathbf{L y s}^{307}(\mathrm{~K})$ in Giardia (Fig. 1d). These lysines are also conserved in other organisms (Fig. 1c) and are involved in binding the substrate phosphate in coordination with $\mathrm{Mg}^{+2}$ [35]. Indeed, a phenylalanine (F) residue is observed upstream the SARM motif in all sequences of different organisms except for Giardia that has a lysine (K) residue (Fig. 1b). Previous work identified seven conserved regions in FPPS from trypanosomatids [19], the seventh region in the C-terminal region in Giardia has a conserved arginine residue and eight extra amino acids (Fig. 1e).

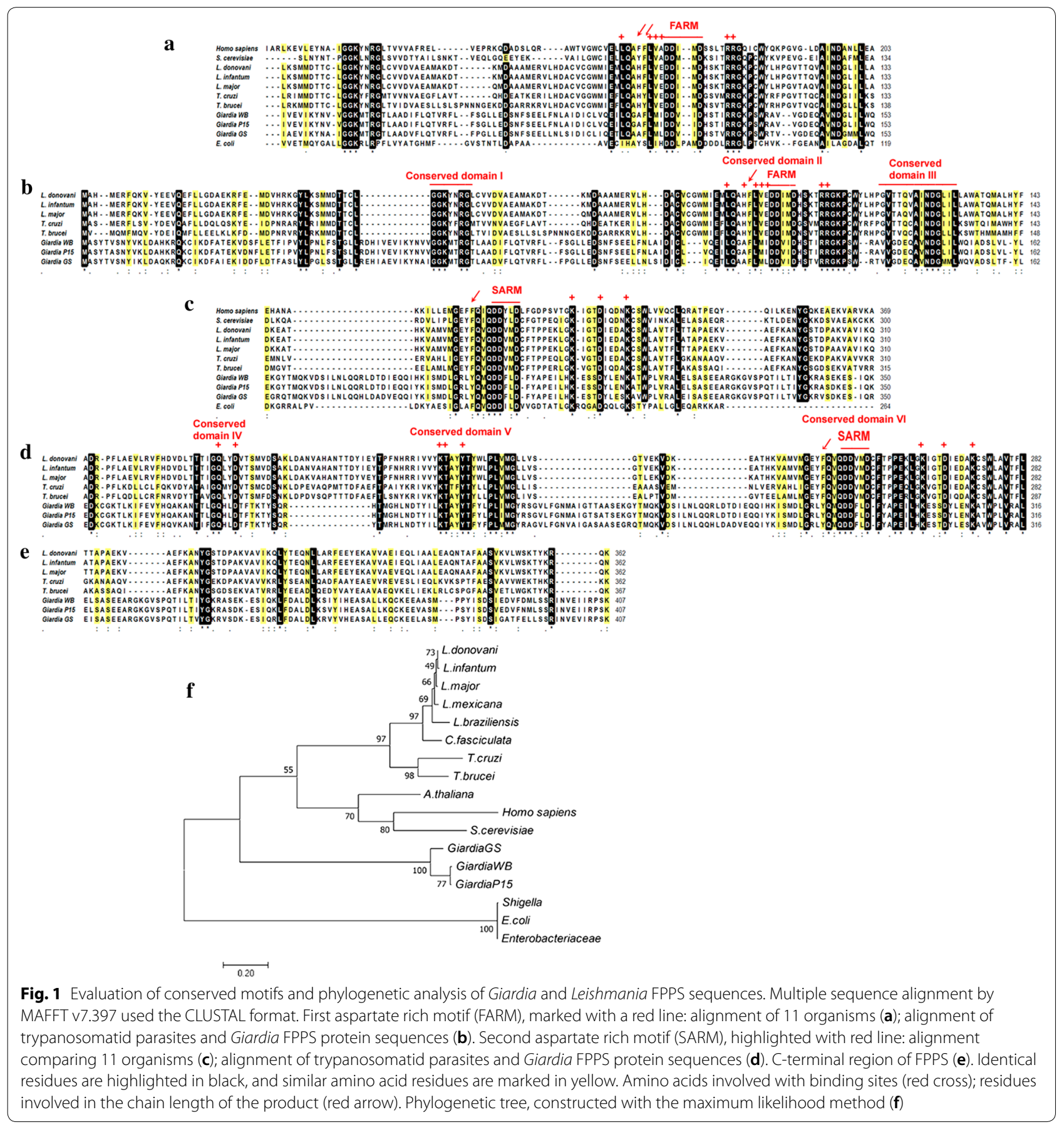




\section{Three-dimensional structure prediction and structural analyses}

Here, the 3D model of the L. infantum FPPS was generated via comparative modeling based on the L. major structure (PDB ID 4JZB). The sequence alignment between L. major and L. infantum FPPS proteins had $96.95 \%$ identity with a coverage of $99 \%$. We chose the model with the lowest DOPE scores (-47931, 92188) among the 50 generated models. The Ramachandran plot of the selected model shows that $100 \%$ of all residues were allocated in energetically allowed regions with 99.2\% in the favored region. The overall quality factor achieved with ERRAT was 92.6554, and the Verify 3D server estimated that $94.20 \%$ of the residues of $L$. major FPPS had an averaged 3D-1D score $\geq 0.2$. These results indicate that the refined model has good quality and is reliable for further computational analysis.

The best results for the Giardia WB FPPS sequence were obtained with PDB 6B02 from a similarity search against PDB sequences using BLASTp [36]. The alignment between the Giardia WB FPPS and the template sequences showed that the identity and coverage were $29.57 \%$ and $81 \%$, respectively. Structural prediction by threading methods was applied due to this low sequence identity with any other organism and the lack of experimentally elucidated three-dimensional structures. The Ramachandran plot of the resulting model had $93.8 \%$ of all residues allocated in energetically allowed regions with $76.2 \%$ in the favored region. The ERRAT analysis showed an overall quality factor higher than 81.7 . Verify3D showed that $65.36 \%$ of the amino acid scored $\geq 0.2$ in the 3D/1D profile. Thus, the Giardia FPPS theoretical model has sufficient quality to perform in silico analysis.

The structures are conserved when analyzing the alignment of the FPPS structures from organisms belonging to the Leishmania genus (Fig. 2a); the RMSD is $0.221 \AA$. The conserved residues found in FARM (DDIMD) and SARM (DDVMD) motifs for L. major and L. infantum are represented in Fig. 2b and c, respectively. However, the structural alignment between the $L$. major crystallographic structure and the G. duodenalis theoretical model displayed a lack of conserved residues that compose the regions outside the FARM and SARM motifs (Fig. 2d-f). The aspartic acid residues presented in FARM and SARM motifs (DDXXD) are part of the catalytic cavity and have an important role in protein function. Meanwhile, the residues represented by XX are the same for $L$. major and L. infantum in FARM (Fig. 2b) and SARM (Fig. 2c) but are different for G. duodenalis FARM (Fig. 2e) and SARM (Fig. 2f) motifs.

Importantly, even if there is a difference between the electrostatic potential on the surfaces of the three-dimensional structures of the FPPS, the site of the FARM and SARM motifs is electrostatically negative (Fig. 2g).

\section{Effect of N-BPs on Leishmania and Giardia}

The expression of sterol biosynthetic enzymes are upregulated in the insect stage of Trypanosoma brucei and Trypanosoma cruzi procyclic and epimastigote forms [3]; thus, we evaluated N-BP inhibition on the promastigotes of L. infantum. Giardia has four enzymes of the MVA pathway: acetoacetyl-CoA thiolase, HMG-CoA-synthase, HMG-CoA reductase, and mevalonate kinase, expressed on the trophozoites stage [37].

We performed at least three assays with each bisphosphonate in the range of $5-500 \mu \mathrm{M}$ on promastigotes of $L$. infantum for $72 \mathrm{~h}$ incubation. We also evaluated the cytotoxic effect of bisphosphonates on G. duodenalis trophozoites and created concentration curves from $10 \mu \mathrm{M}$ to $1 \mathrm{mM}$ for $48 \mathrm{~h}$ of incubation. Risedronate, ibandronate, and alendronate have increased antiproliferative activity on promastigotes of L. infantum (Table 2) versus trophozoites of G. duodenalis (Table 2). Only risedronate and ibandronate display antiproliferative activity (Table 2) in G. duodenalis as evaluated by the $\mathrm{IC}_{50}$.

\section{Effect of N-BPs on the ultrastructure of Leishmania and Giardia}

Leishmania promastigotes treated with risedronate, ibandronate, and alendronate displayed the same ultrastructural alterations. To correlate the ultrastructural alterations caused by N-BPs with mechanisms of cell death in Leishmania, we evaluated the L. infantum promastigotes treated with $100 \mu \mathrm{M}$ alendronate or $20 \mu \mathrm{M}$ risedronate. Promastigotes accumulated small vesicles in the Golgi region near the kinetoplast (Fig. 3d, e) as well as mitochondrial swelling (Fig. 3e, k), altered cell division (Fig. 3h), formation of intracellular vesicles and lamellae (Fig. 3f-j), blebbing of the plasma membrane (Fig. 3g), as well as nuclear pyknosis and chromatin condensation (Fig. 3f, j). There was also an invagination of the plasma membrane in the flagellar pocket region without membrane rupture (Fig. 3i, j) as well as concentric membranes in regions of the mitochondria and myelin figures (Fig. 3k, l). Membrane integrity can distinguish apoptosis from necrosis. Promastigotes treated with alendronate have preserved plasma and nuclear membranes, as evaluated by electron microscopy.

Giardia duodenalis treated with $300 \mu \mathrm{M}$ risedronate or ibandronate for $48 \mathrm{~h}$ had a high frequency of concentric membranes near the nuclei as well as nuclear pyknosis and membrane layers and lamella formation on the nucleus (Fig. 4d, g, h). There was membrane detachment and formation of intracellular lamellae in the cytoplasm 


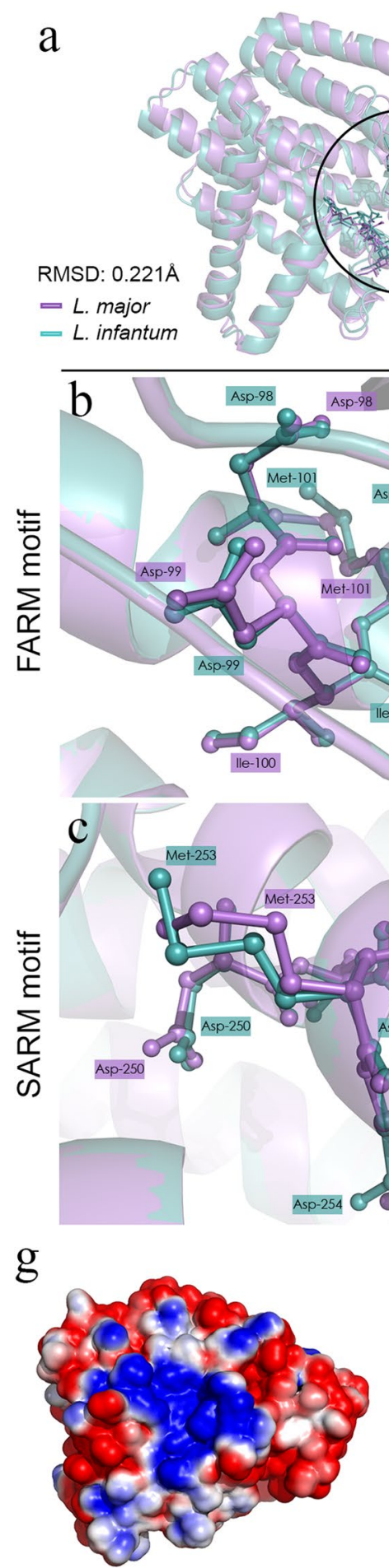

L. major

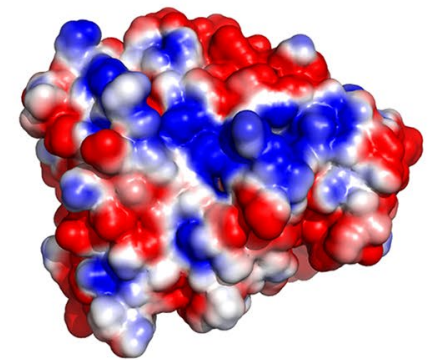

L. infantum
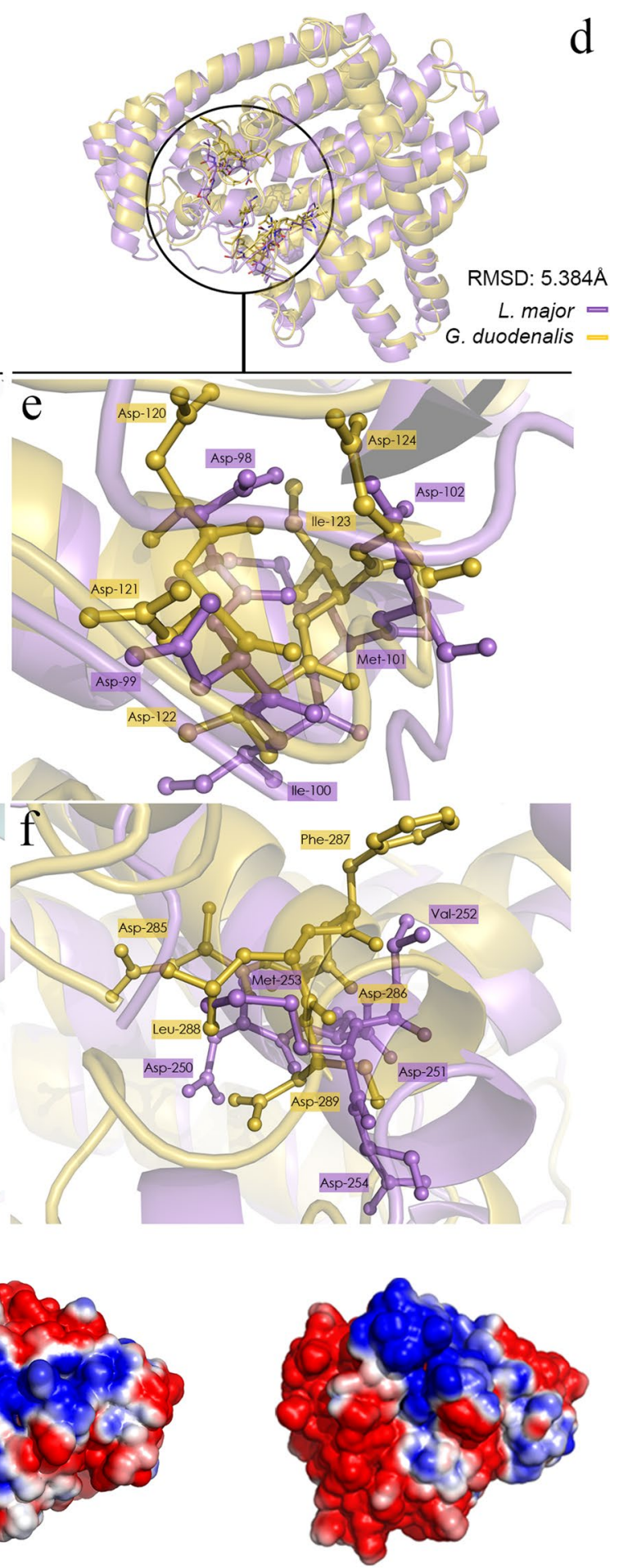

G. duodenalis

Fig. 2 Structure comparison between FPPS of Leishmania and Giardia. Three-dimensional structure alignments based on the structural similarity of L. major FPPS crystallographic structure (PDB 4JZB, lilac chain A) with L. infantum (green) (a) and alignment of L. major FPPS (lilac) with G. duodenalis (yellow) (d). The inset highlights the residues (sticks) of FARM ( $\mathbf{b}, \mathbf{e})$ and SARM $(\mathbf{c}, \mathbf{f})$ motifs in the catalytic site. Residues of both motifs, FARM and SARM, are conserved in genus Leishmania $(\mathbf{b}, \mathbf{c})$, and the residues of this region are not conserved between L. major and G. duodenalis (e, $\mathbf{f})$. Electrostatic potential surface map generated by PyMol for the structures of FPPS $(\mathbf{g})$. The color scale for surface electrostatic potential was set from $-1 \mathrm{kT} / \mathrm{e}$ (red) to $1 \mathrm{kT} / \mathrm{e}$ (blue). Key: white, neutral; blue, positive charge; red, negative charge 
(Fig. 4d-f). Small vesicles were distributed in the cytoplasm (Fig. 4e). Intense myelin figures suggested nuclear engulfment (Fig. 4i), which can be caused by membrane accumulation from the endoplasmic reticulum. These ultrastructural alterations are due to disorganization of the endomembrane system.

\section{Evaluation of programmed cell death in Leishmania treated with N-BP}

We evaluated apoptosis and necrosis caused by N-BPs with three probes: (i) Annexin-V-FITC that labels exposed phosphatidylserine; (ii) 7-amino-actinomycin D (7-AAD) to evaluate plasma membrane integrity; and (iii) TMRE to estimate the loss of mitochondrial potential. A negative control comprised of viable promastigotes without N-BPs treatment was not stained with Annexin-VFITC and/or 7-AAD. Control promastigotes after $72 \mathrm{~h}$ of cultivation did not undergo the process of apoptosis or necrosis (Fig. 5a).

The $L$. infantum promastigotes treated with $10 \mu \mathrm{M}$ miltefosine for 48 or $72 \mathrm{~h}$ had two populations of promastigotes that increased with time as seen in the SSC $\times$ FSC plot (Fig. 5b). After $72 \mathrm{~h}$ of incubation with miltefosine, population 1 displayed co-staining with annexin- $\mathrm{V}$ and 7-AAD or just 7-AAD staining, and population 2 was not stained or displayed only annexin- $V$ staining. Incubation of $L$. infantum promastigotes with $10 \mu \mathrm{M}$ miltefosine induced co-staining in $12 \%$ and $31 \%$ after 48 and $72 \mathrm{~h}$, respectively. Staining with only annexin-V was reported in $9.2 \%$ and $8.9 \%$ and staining with $7-\mathrm{AAD}$ was observed in $1.7 \%$ and $21 \%$ of promastigotes. These results indicate that miltefosine caused apoptosis and necrosis.

Promastigotes treated with $100 \mu \mathrm{M}$ alendronate for 24, 48 and $72 \mathrm{~h}$ (Fig. 5c) displayed Annexin-VFITC staining in $22.8 \%, 36 \%$ and $34 \%$ of promastigotes, respectively. Co-staining with annexin-V-FITC and 7 -AAD was observed in $30 \%, 24 \%$ and $25 \%$ of the population. There was low staining with 7 -AAD; $0.8 \%$ to $1.3 \%$ after 24 to $72 \mathrm{~h}$. The high percentage of single

Table 2 Effect of FPPS inhibitors on trophozoites of Giardia duodenalis and on promastigotes of Leishmania infantum

\begin{tabular}{lll}
\hline $\begin{array}{l}\text { Nitrogen } \\
\text { bisphosphonates (N-BP) }\end{array}$ & \multicolumn{1}{l}{. 5 Mean \pm SD $(\mu \mathrm{M})$} & \\
\cline { 2 - 3 } & L. infantum & G. duodenalis \\
\hline Risedronate & $13.8 \pm 6.0(n=5)$ & $311 \pm 120(n=3)$ \\
Ibandronate & $85.1 \pm 26.5(n=4)$ & $271 \pm 62(n=3)$ \\
Alendronate & $112.2 \pm 61.2(n=5)$ & nd \\
Pamidronate & $118.7 \pm 26.2(n=2)$ & nd \\
Neridronate & $173.3 \pm 31.6(n=3)$ & nd \\
\hline
\end{tabular}

Note: $\mathrm{IC}_{50}$ was estimated by the MTS method labelling with annexin-V indicates exposure of PS and early apoptosis. The co-staining and the nearly absent labelling of promastigotes with 7-AAD alone reflects apoptosis in an advanced stage in L. infantum promastigotes. The dispersion plot $(\mathrm{SSC} \times \mathrm{FSC})$ shows that promastigotes treated with alendronate have two populations. The cells with volume preserved (over 60\% of the total) were composed of unlabeled promastigotes or those only labelled with annexin-V (Fig. 5c). The second population was comprised mainly of promastigotes co-stained with annexin- $\mathrm{V}$ and 7AAD and represents damaged promastigotes.

We included controls comprising Annexin-V-FITCand 7-AAD-labelled promastigotes of Leishmania permeabilized with Triton X-100. The forward and side scatter plot (Fig. 5d) demonstrated a loss of membrane integrity and cellular volume. As expected, co-staining with annexin-V-FITC and 7-AAD was observed in $66-74 \%$ of promastigotes (Fig. 5 d).

\section{Evaluation of mitochondria membrane potential damage in Leishmania treated with N-BP}

We also evaluated the mitochondrial potential with tetramethylrhodamine ethylesterpercholate (TMRE) in the same culture of $L$. infantum promastigotes treated for $72 \mathrm{~h}$ with N-BPs or miltefosine. TMRE is a cationic lipophilic dye that accumulates in the active mitochondrial of viable protozoans; the fluorescent intensity is a direct measure of its accumulation and cellular metabolism. We evaluated $L$. infantum promastigotes untreated with drugs and unlabeled with TMRE, as control, by means of a light scattering plot (SSC $\times$ FSC) (Fig. 6a) and FL2 (Fig. 6b). The evaluation of mitochondrial function with TMRE (FL2) displayed that $93.6 \%$ of the promastigotes incorporated TMRE, represented by M2. The M2 population displayed two contiguous peaks: one had higher TMRE incorporation and was represented by $25.5 \%$ of the promastigotes; and the second one is composed of $68.5 \%$ (Fig. 6c). This second group is likely promastigotes in stationary phase, they had reduced division and mitochondrial activity. Similarly, two populations of promastigotes from $L$. donovani were observed with TMRE and increased after 6 and 7 days of cultivation [38].

After incubation with miltefosine and alendronate, another population with low or absent TMRE incorporation appeared and was represented by M1. This population increased with miltefosine concentration from 5 to $10 \mu \mathrm{M}$ (not shown). The dispersion plot $(\mathrm{SSC} \times \mathrm{FSC})$ demonstrated two populations of promastigote (Fig $6 \mathrm{~d}, \mathrm{~g}$ ) indicative of promastigotes with reduced cellular volume and membrane damage. After incubation with TMRE, M1 represented $51.1 \%$ of promastigotes affected by 10 $\mu \mathrm{M}$ miltefosine under apoptosis or necrosis, and M2 

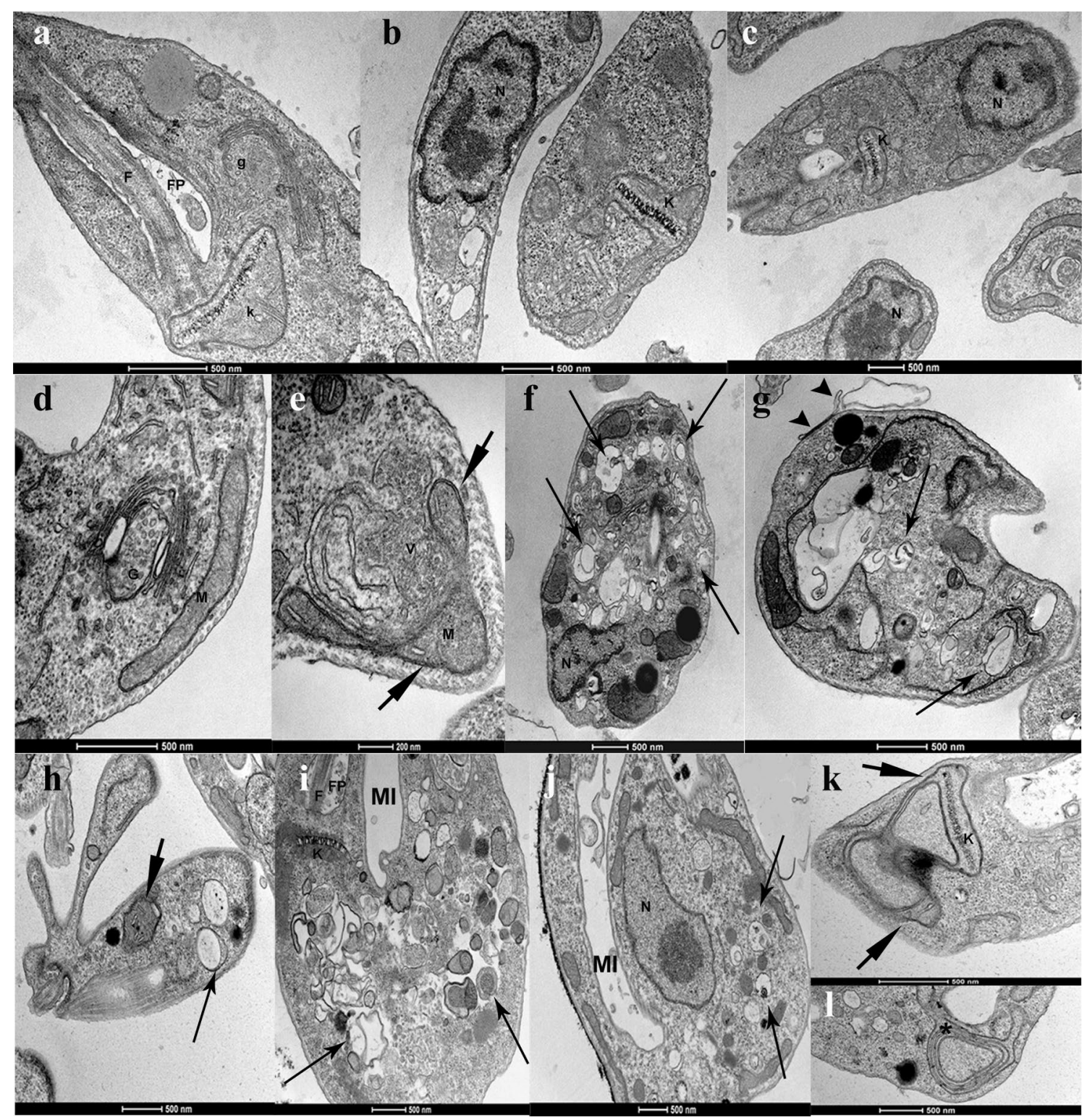

Fig. 3 Ultrastructural evaluation of L. infantum treated with N-BP. Promastigotes of L. infantum: control, not treated (a-c); treated with risedronate $(\mathbf{d}-\mathbf{g})$; treated with alendronate $(\mathbf{h}-\mathbf{I})$. Mitochondrial swelling (short arrow); double membrane and vacuoles (long arrow); myelin figure $(*) ;$ plasma membrane blebs (arrowhead). Abbreviations: N, nucleus; K, kinetoplast, M mitochondria; F flagellum; FP, flagellar pocket; G, Golgi; MI, membrane invagination. Scale-bars: a-d, $500 \mathrm{~nm} ; \mathbf{e}, 200 \mathrm{~nm} ; \mathbf{f}-\mathbf{I}, 500 \mathrm{~nm}$

represented promastigotes with functional mitochondria (45.9\%; Fig. 6e). The overlay (Fig. 6f) of the TMRE positive control (Fig. 6c) versus promastigotes treated with miltefosine (Fig. 6e) showed a decrease of the M2 subpopulation.

Alendronate $(100 \mu \mathrm{M})$ abolished mitochondrial membrane potential but in a lower percentage of promastigotes than miltefosine. In our assays, $100 \mu \mathrm{M}$ alendronate could reduce mitochondrial membrane potential in approximately $28 \%$ of promastigotes as represented by
M1 (Fig. 6h). The M2 population predominated in promastigotes treated with alendronate (71.3\%). An overlay of the histograms shows that the positive control with TMRE and treated with alendronate for $72 \mathrm{~h}$ had a M1 population with slight displacement of M2 to the lower fluorescent emission indicating lower TMRE labeling (Fig 6i). As expected, the same population labelled with annexin- $\mathrm{V}$ and 7-AAD had a loss of mitochondrial functions. 


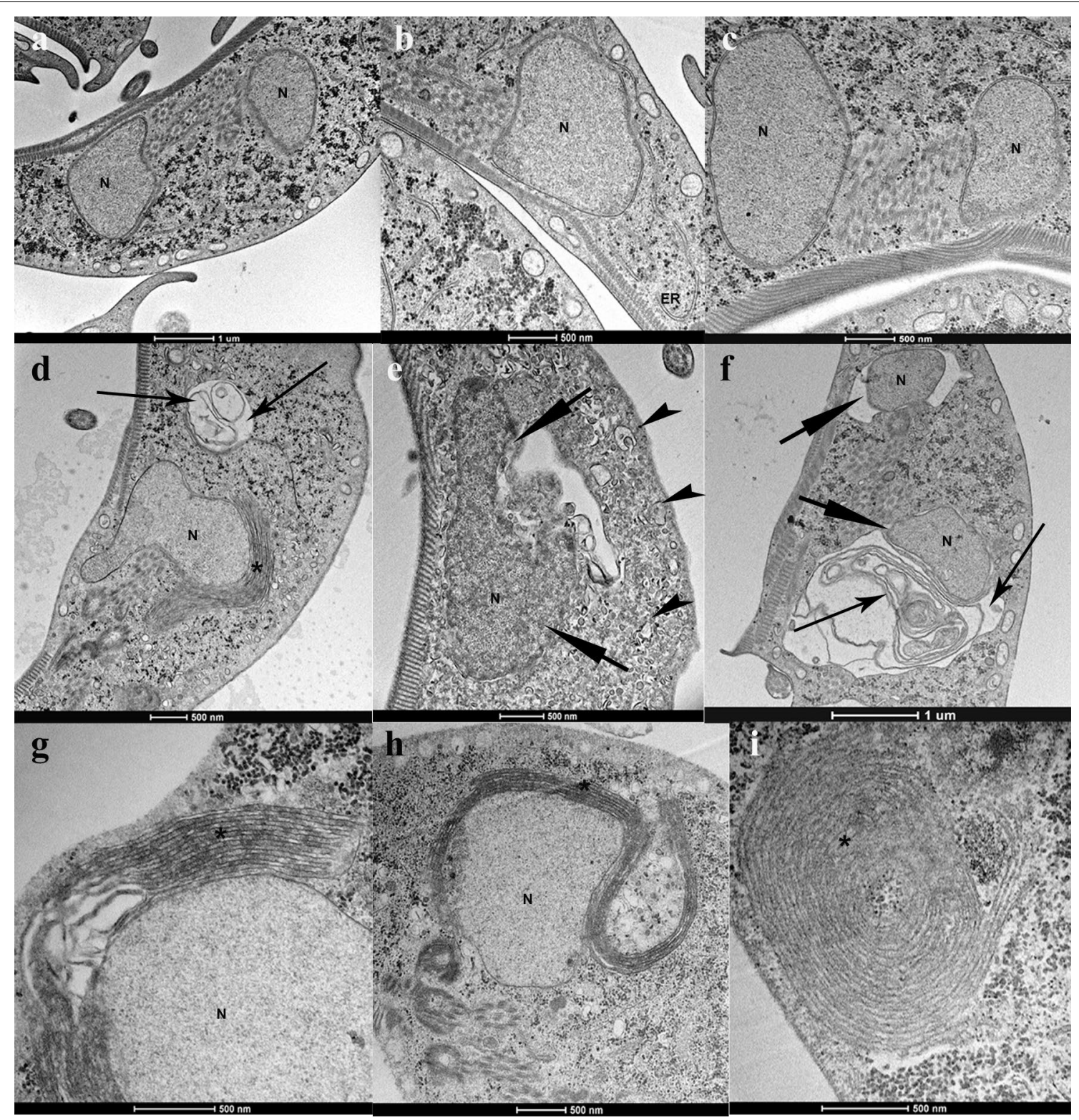

Fig. 4 Ultrastructural evaluation of Giardia treated with N-BP. Trophozoites of G. duodenalis: control, not treated (a-c); treated with risedronate (d-f); treated with ibandronate $(\mathbf{g}-\mathbf{i})$. Nuclear pyknosis (short arrow); double membrane, intracellular vesicles, and lamellae (long arrow); small vesicles

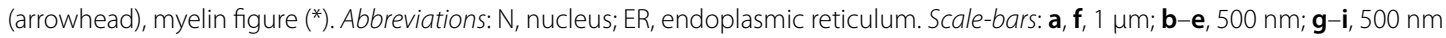

\section{Discussion}

A previous report compared trypanosomatids with apicomplexans by multiple sequence alignment and predicted seven conserved domains in the FPPS enzyme
[19]. We highlighted these conserved domains in the alignment of Leishmania FPPS with different Giardia assemblages, and FPPS sequences from other organisms (Fig. 1a, c). The Giardia FPPS enzyme possesses

(See figure on next page.)

Fig. 5 Analysis of cell death in promastigotes of $L$. infantum treated with alendronate. Negative control: promastigotes not treated with alendronate and stained with Annexin $\mathrm{V}$ and 7-AAD (a). Promastigotes treated with $10 \mu \mathrm{M}$ miltefosine for 72 hours and stained with Annexin V and 7-AAD (b). Promastigotes treated with $100 \mu \mathrm{M}$ alendronte for 72 hours and stained with Annexin $V$ and 7-AAD (c). Promastigotes permeabilized with triton X-100, 0.5\%, and stained with Annexin V and 7-AAD (d). SSC versus FSC (left panels); and FITC Annexin V (FL1) versus 7-AAD (FL3) (right panels) 

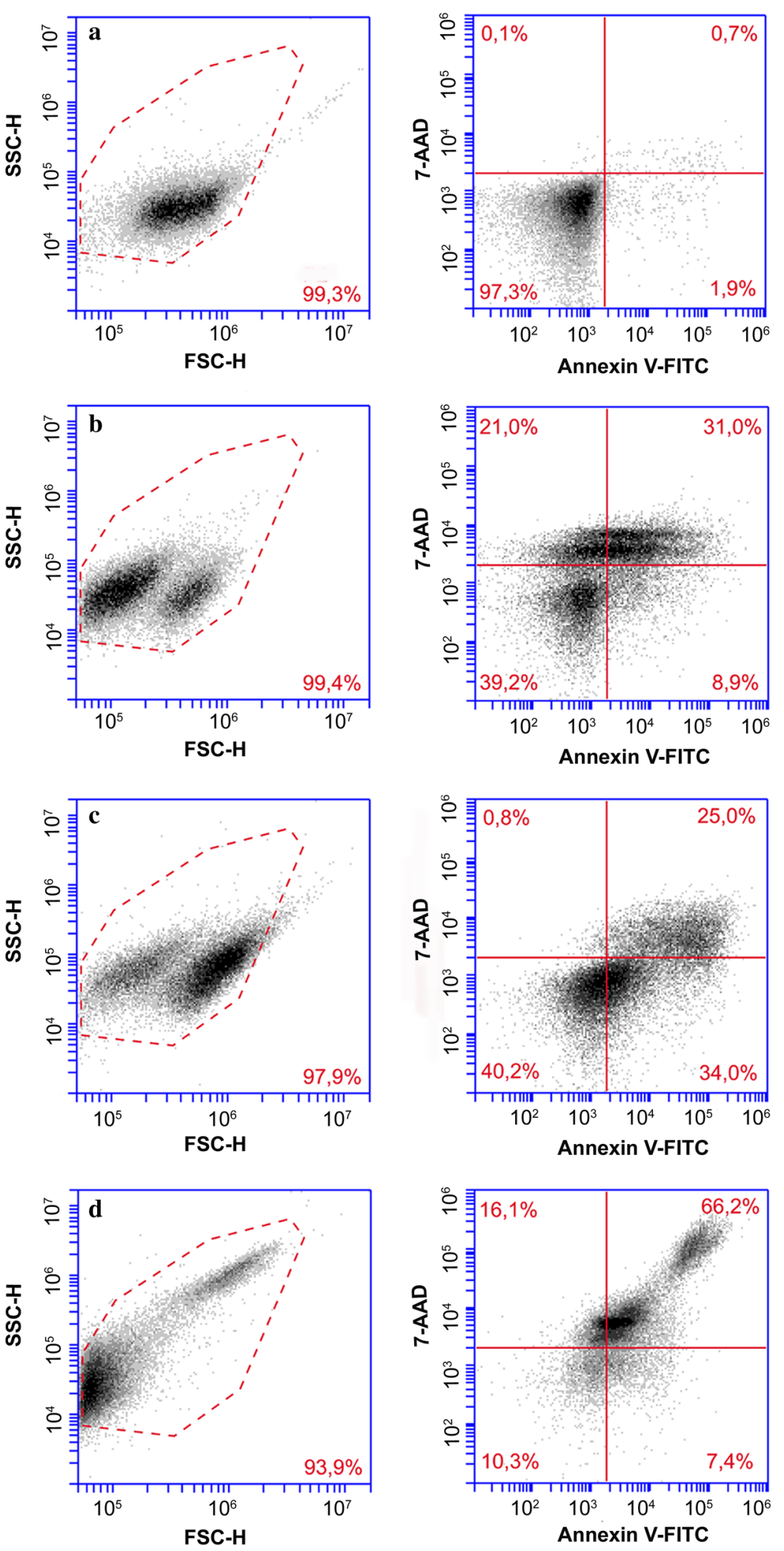

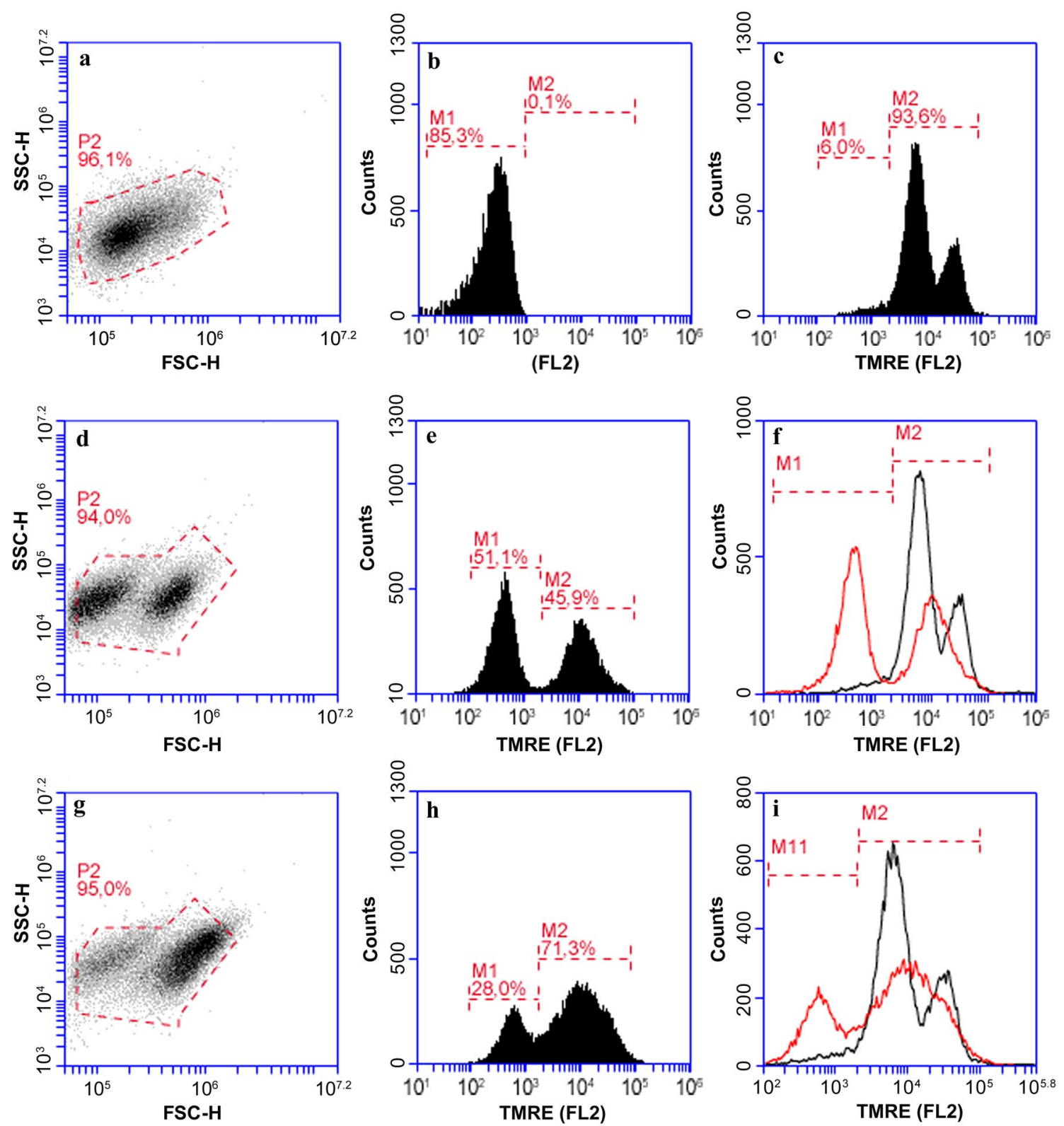

Fig. 6 Analysis of mitochondrial membrane potential in promastigotes of L. infantum treated with alendronate. Negative control: promastigotes not treated with drugs and not stained with TMRE: light scattering plot shows one population (a) with low fluorescence intensity (M1) (b). Positive control, promastigotes not treated and stained with TMRE possesses high fluorescence (M2 peaks) (c). Treated with $10 \mu \mathrm{M}$ miltefosine for $72 \mathrm{~h}$ and stained with TMRE: light scattering plot shows two populations (d) with decreased M2 coupled to an increase in the M1 population (e). Treated with $100 \mu \mathrm{M}$ alendronate for $72 \mathrm{~h}$ and stained with TMRE: light scattering plot shows two populations (g) with; slight decrease in M2 population to M1 region (h). Overlay of positive control (black histogram) with drug-treated parasites (red histogram) (f, i)

405 amino acids, which is about 40 amino acids longer than FPPS from Leishmania and trypanosomatids: this is reflected in long internal loops and the carboxyl terminal region (Fig. 1e). The human FPPS carboxy terminal tail has a conserved domain VII and adopts a rigid configuration. The conserved $\mathrm{Arg}^{351}$ side chain forms a bridge with the terminal $\operatorname{Lys}^{353}(\mathrm{~K})$. There are amino acid residues that switch on and off the tail configuration [39]. Leishmania major and T. cruzi FPPS lack this mechanism. However, Giardia has 8-mers increase in the carboxyl terminal tail with a possible similar role.

Despite the differences between Leishmania and Giardia FPPS sequences, the main FPPS signatures were identified in Giardia sequences including the FARM 
motif. This motif allows the classification of FPPS in type I enzymes (eukaryotic origin) or type II enzymes (prokaryotic origin). Type I FARM has a DDXXD signature found in trypanosomatids, S. cerevisiae, H. sapiens and in Giardia (Fig. 1b), type II displays two extra amino acids inside the FARM sequence, i.e. DDXXXXD sequence [40, 41], as demonstrated in E. coli (Fig. 1a). Previous studies demonstrated that aromatic or bulky amino acids at the fourth to fifth amino acids upstream of the FARM motif are fundamental for product specificity and length. They are another characteristic of type I enzymes.

Figure 1a shows E. coli FPPS, a type II enzyme without these residues before the FARM motif. These residues are also involved in the binding of NB-P inhibitors and interactions with the phenyl ring of bisphosphonates [41]. The aspartate-rich domains have a role in substrate binding through the phosphates in coordination with $\mathrm{Mg}^{+2}$ ions. The carboxyl group of aspartate, residues 98-102 of FARM, and residues 250-254 of SARM coordinate with the phosphate atoms inside the catalytic pocket. The phosphates of N-BP also make hydrogen bonds with the amino groups in Lys $^{207}$ and Lys ${ }^{264}[20,35]$.

Human FPPS has two phenylalanine residues (Fig. 1a) at the fifth and fourth positions upstream of the FARM motif [20]. In L. major, $\mathrm{His}^{93}(\mathrm{H})$ replaces the phenylalanine at the fifth position, and there is a $\mathrm{Phe}^{94}$ at the fourth position. In T. cruzi FPPS, both phenylalanines are replaced, the fifth one by $\operatorname{His}^{93}(\mathrm{H})$, and the fourth by the aromatic amino acid $\operatorname{Tyr}^{94}(\mathrm{Y})[20,21]$. In Giardia, the fifth phenylalanine upstream the FARM motif is replaced by $\mathrm{Ala}^{115}(\mathrm{~A})$, but the $\mathrm{Phe}^{116}(\mathrm{~F})$ at the fourth position is maintained. Phenylalanine and tyrosine can limit prenyl chain elongation. Amino acid residues with a smaller side chain (alanine or glycine) can impact the product specificity and increase the space inside the pocket to accommodate longer length prenyl chains. Thus, functional characterization of Giardia FPPS, i.e. cloning, mutagenesis and kinetic analysis, can elucidate the amino acids that are involved in the affinity for the substrates, for inhibitors (NB-P), and the prenyl chain product.

Previous work demonstrated that FPPS from Giardia formed a separate branch distinct from trypanosomatids (kinetoplastids) and prokaryotes [19], but phylogenetic analysis and multi alignment comparing Giardia assemblages with Leishmania FPPS proteins were not performed. Thus, we constructed a phylogenetic tree of FPPS enzymes from 17 organisms (Fig. 1f): Giardia and Leishmania FPPS sequences were placed in distinct clades.

Molecular modelling of Giardia FPPS using a threading approach was performed due to the low identity of FPPS from Giardia with FPPS from Leishmania and other organisms. The model generated from the enzymes helps to explain the conformation adopted by the Giardia FPPS including the position of the amino acid residues inside and surrounding the FARM motif and the SARM motif (Fig. 2). These are involved in the binding of the substrate and the inhibitors.

Some studies have tested the activity of N-BPs in different protozoans: in vitro assays of T. cruzi and L. donovani amastigotes inside infected Vero cells, T. brucei trypomastigotes, Toxoplasma gondii tachyzoites, and Plasmodium falciparum intraerythrocytic stages. All models demonstrated that N-BPs, especially aromatic compounds such as risedronate, have significant antiprotozoal activity with an $\mathrm{IC}_{50}$ in the nanomolar or low micromolar range [42]. The $\mathrm{IC}_{50}$ for N-BPs for intracellular amastigotes of $T$. cruzi was $147 \pm 31.2 \mu \mathrm{M}$ for alendronate and $123 \pm 26.4 \mu \mathrm{M}$ for risedronate. In amastigotes of $L$. donovani, $\mathrm{IC}_{50}$ for N-BPs was $82.5 \pm 14.6 \mu \mathrm{M}$ for alendronate and $2.3 \pm 0.3 \mu \mathrm{M}$ for risedronate. This demonstrates that the aromatic N-BP, risedronate, has a better activity in $L$. donovani amastigotes.

Promastigotes of Leishmania spp. are good models to evaluate inhibition of the sterol pathway including mechanisms of cell death. They can be used to evaluate the damage and impact on the parasite ultrastructure due to N-BPs and other inhibitors. Despite being the form found in the insect, the dividing promastigotes are easy to cultivate, have active mitochondria, and display high expression of FPPS as evaluated by mRNA and polycistronic RNA [21]. Our study used promastigotes of $L$. infantum and showed that risedronate had higher activity $(13.8 \pm 6.0 \mu \mathrm{M})$ followed by ibandronate $(85.1 \pm 26.5 \mu \mathrm{M})$ and alendronate $(112.2 \pm 61.2 \mu \mathrm{M})$ (Table 2), without toxicity to the host cells as evaluated in RAW and LLCMK2 cells (data not shown).

$\mathrm{N}$-BPs are strong inhibitors of the recombinant FPPS enzyme. N-BPs such as risedronate, alendronate, and pamidronate are competitive inhibitors of IPP and GPP, substrates of FPPS. The recombinant FPPS of T. cruzi displayed a higher affinity for risedronate with a $K_{i}$ of $0.032 \mu \mathrm{M}$ than alendronate and pamidronate $\left(K_{i}\right.$ of $1.04 \mu \mathrm{M}$ and $2.02 \mu \mathrm{M}$, respectively) [22] as estimated by a Dixon plot. Furthermore, when the affinity for each $\mathrm{N}$-BPs was evaluated by the $\mathrm{IC}_{50}$, human recombinant FPPS displayed higher affinity for risedronate with an $\mathrm{IC}_{50}$ of $0.010 \mu \mathrm{M}$ versus T. cruzi recombinant FPPS with an $\mathrm{IC}_{50}$ of $0.037 \mu \mathrm{M}$ for risedronate [22]. Leishmania major recombinant FPPS enzyme also showed higher activities for risedronate $\left(\mathrm{IC}_{50}\right.$ of $\left.0.17 \mu \mathrm{M}\right)$ than ibandronate $\left(\mathrm{IC}_{50}\right.$ of $\left.0.48 \mu \mathrm{M}\right)$ [21]. These differences in the $\mathrm{IC}_{50}$ of the FPPS recombinant enzymes from different organisms can be associated with the amino acid residues found in the binding pocket surrounding the FARM and 
SARM motifs; these can alter the affinity for the N-BP inhibitors.

Our electron microscopic data showed that N-BPs causes several alterations in intracellular membrane and organelles of Leishmania such as myelin figures, mitochondrial swelling, plasma membrane blebs and membrane disorganization (Fig. 3). Previous studies demonstrated that $L$. amazonensis treated with specific inhibitors of ergosterol biosynthesis display morphological alterations and cell death associated with sterol depletion [2]. We also observed Golgi disorganization with small vesicles distributed in the cytoplasm as well as invagination near the flagellar pocket (Fig 3); these are suggestive of alterations in the exocytosis and endocytosis. The inhibition of protein prenylation by bisphosphonates, inhibitors of prenyl protein transferases, or inhibitors of mevalonate or isopentenyl pyrophosphate synthesis (lovastatin, mevastatin and phenylacetate) can profoundly affect cell morphology, cell replication, intracellular signal transduction, and lead to cell death by apoptosis, as demonstrated elsewhere [22, 24].

Programmed cell death was described previously in trypanosomatids and in $L$. donovani promastigotes and amastigotes, caused by parasites in stationary phase or induced by pentostan and amphotericin B. These drugs can induce PPL-cleavage activity, change membrane integrity, increase the electron density in the cytoplasm, and lead to nuclear condensation [38]. Previous publications describing T. cruzi epimastigotes treated with the sterol biosynthesis inhibitors, ketoconazole and lovastatin, indicated branching of the mitochondrial membranes including a concentric pattern of the inner mitochondrial membrane in contact with kinetoplast and myelin figures suggestive of autophagy [43].

There are also reports of increased intensity in the membrane potential after rhodamine treatment [43]. In contrast, we did not observe increased mitochondrial potential, as evaluated via TMRE nor branching of mitochondrial membranes in promastigotes by electron microscopy after treatment with N-BP.

Our results suggest that necrosis is not the main mechanism of $L$. infantum promastigotes death caused by N-BPs, only $\sim 1 \%$ of the promastigotes were stained with 7-AAD alone after 48 to $72 \mathrm{~h}$ incubation with alendronate (Fig. 5). Indeed, staining with Annexin-V alone and co-staining with 7-AAD led to $34 \%$ and $25 \%$ labelling, respectively (Fig. 5), and the co-stained population had a loss of membrane potential (TMRE) (Fig. 6). In contrast, T. cruzi epimastigotes treated with ketoconazole and lovastatin for $12 \mathrm{~h}$ had marked co-staining with Annexin-V and PI or with PI alone, and very little Annexin-V staining [43].
Instead, we suggest that apoptosis is the main mechanism of death caused by N-BPs in L. infantum promastigotes. It was described that inhibition of protein prenylation by N-BP is one of the main mechanisms underlying decreased bone resorption by osteoclasts. Apoptosis has been reported for osteoclasts treated with N-BPs [44]. Indeed, N-BPs such as clodronate, etidronate, pamidronate, alendronate, and risedronate in concentrations of 10 and $1000 \mu \mathrm{M}$ induced apoptosis in Caco-2 human epithelial cells [45]. A recent in vivo study demonstrated that subcutaneous administration of zoledronic acid in mice inhibits prenylation of Rab1A, Rab5B, Rab7A, and Rab14 in mouse peritoneal macrophages [46].

Ubiquinone or coenzyme $\mathrm{Q}(\mathrm{CoQ})$, a component of the electron transport chain in aerobic organisms as Leishmania, can have biosynthesis affected by inhibition of FPPS due to N-BPs, which can be correlated also with the mitochondrial damage observed by electron microscopy in the promastigotes of $L$. infantum treated with risedronate and alendronate. Depending on the Leishmania species and life stage, coenzyme CoQ8 and CoQ10 were detected in lower amounts. CoQ9 is the predominant homologue, and it has been detected in all organisms including ones without identifiable mitochondria such as Giardia, which has only a reminiscent mitochondrion [15].

The N-BPs activity was more pronounced in L. infantum than in trophozoites of G. duodenalis, as evaluated by the viability method. We found an $\mathrm{IC}_{50}$ of $271 \pm 62 \mu \mathrm{M}$ for ibandronate and $311 \pm 120 \mu \mathrm{M}$ for risedronate (Table 2). The higher activity, anti-proliferative effect of N-BPs, such as risedronate on L. infantum, compared to Giardia, can relate to the fact that Giardia has a minimal sterol metabolism, and to the differences in the catalytic site and pocket of the FPPS in each organism.

The inhibitory concentration of risedronate and ibandronate N-BP was higher in Giardia trophozoites than Entamoeba indicating a lower activity versus Entamoeba. Previous studies evaluated the N-BP activity in amitochondriate Entamoeba histolytica, compared to the apicomplexan parasite Plasmodium. Trophozoites of E. histolytica and $P$. falciparum in the intraerythrocytic stage displayed an $\mathrm{IC}_{50}$ above $200 \mu \mathrm{M}$ for alendronate and pamidronate [47]. The $\mathrm{IC}_{50}$ values were $73.5 \mu \mathrm{M}$ and $123 \mu \mathrm{M}$ for risedronate and $53.6 \mu \mathrm{M}$ and $50.1 \mu \mathrm{M}$ for ibandronate, respectively [47]. In Giardia and E. histolytica, inhibition of FPPS by N-BP can impact the biosynthesis of dolichol and isoprenoids because ergosterol biosynthesis is absent.

Another aspect to consider is the expression of FPPS in different subcellular compartments, affecting the N-BP intracellular distribution. An FPPS fusion with GFP 
demonstrated localization in peroxisomes in the amoeba Dictyostelium discoideum [48]. An enzyme in mevalonate pathway, 3-hydroxy-3-methylglutaryl-coenyzme A (HMG-CoA) reductase, is an integral enzyme of endoplasmic reticulum (ER) in the amitochondriate Giardia. The ER membranes are the site of polyisoprenoids and dolichol biosynthesis.

Trophozoites of G. duodenalis treated with N-BPs displayed many more myelin figures (Fig. 4) than L. infantum promastigotes. Concentric membranes around the nucleus and around other organelles are indicative of autophagy. The main pathways affected by inhibition of FPPS are the prenylation of proteins and dolichols biosynthesis. In accordance with previous biochemical evidence involving the incorporation of labeled FPP and GGPP isoprenoids in GTP binding proteins, Giardia performs isoprenylation of 50 and $21-26 \mathrm{kDa}$ proteins [49]. Prenylation is essential for GTP-binding proteins function, because it is required for protein association to intracellular membranes and for protein-protein interactions including intracellular vesicular transport, membrane endocytosis, and exocytosis.

\section{Conclusions}

Inhibition of the enzyme FPPS by N-BPs can cause a shortage of GPP, FPP and GGPP, which are intermediate metabolites involved in the regulation of cellular functions and homeostasis. A shortage of FPP can cause failure in the isoprenylation of proteins as well as the nuclear lamina and Rab GTPases that are anchored in the intracellular region of the plasma membrane. The nuclear lamina and Rab GTPases interfere with the vesicular transport, endocytosis and exocytosis. Deficits in the synthesis of dolichol interfere with asparagine $(\mathrm{N})$-linked glycosylation that regulates numerous cellular activities such as glycoprotein quality control, intracellular trafficking and cell-cell communications. These alterations concur with our findings; disorganization of intracellular membranes culminating in Leishmania apoptosis. The inhibition caused by NB-P in promastigotes of Leishmania and on trophozoites of Giardia suggests that they are good models to evaluate protein prenylation and mechanisms of cell death. FPPS is in a branching point in sterol metabolic pathways. It is a key enzyme in the mevalonate pathway and a good candidate for drug design. Based on the catalytic site and mechanism of catalysis of the FPPS in each organism, it is possible to develop specific bisphosphonate inhibitors with high affinity for FPPS expressed in each protozoan.

\section{Abbreviations}

FPPS: farnesyl pyrophosphate synthase; GGPPS: geranylgeranyl diphosphate synthase; N-BP: nitrogen-containing bisphosphonate; IPP: isopentenyl diphosphate; DMAPP: dimethylallyl diphosphate; FPP: farnesyl diphosphate; GGPP: geranylgeranyl diphosphate; NCBI: National Center for Biotechnology Information; BLAST: Basic Local Alignment Search Tool; PDB: protein data bank; MAFFT: multiple alignment using fast Fourier transform; MTS: tetrazolium 3-(4,5-dimethyl-2-thiazolyl)-5-(3-carboxymethoxyphenyl)-2-(4-sulfophenyl)-2H; PMS: 5-methyl-phenazinium methyl sulfate; 7-AAD: 7-amino-actinomycin; TMRE: tetramethylrhodamine ethyl ester; SBG: $21 \mathrm{mM}$ Hepes, $0.7 \mathrm{mM} \mathrm{Na} 2 \mathrm{PO}_{4}$, $137 \mathrm{mM} \mathrm{NaCl}, 5 \mathrm{mM} \mathrm{KCl}, \mathrm{pH}$ 7.4, supplemented with $6 \mathrm{mM}$ glucose.

\section{Acknowledgements}

We are grateful to Drs. Maria de Fátima Cepa Matos, Alda Maria Teixeira Ferreira and Inês Aparecida Tozetti for the scientific discussion and the use of microplate reader, Dr. Marcos Paulo Catanho, IOC-Fiocruz, for the discussion in bioinformatics and Rachel Rachid of CENABIO III (Centro de microscopia, Instituto Nacional de Ciência e Tecnologia de Biologia Estrutural e BioimagemUFRJ) for assistance with the electron microscopy.

\section{Authors' contributions}

APRG performed the sample processing for electron microscopy evaluation, the electron microscopy acquisition and drafting the manuscript. MBS and CMB performed the viability assays, and sample processing for electron microscopy. WS designed the experiments for electron microscopy, evaluated the results and peformed the English correction of the manuscript. FP designed the viability assays, corrected and prepared the manuscript. ABMR and ACRG, performed in silico analysis, the theoretical model of FPPS. CH, supervisor of CMB project coordinator from FUNDECT, designed experiments and bioinformatic analysis. All authors read and approved the final manuscript.

\section{Funding}

Financial support for this research was provided by Fundação de Apoio ao Desenvolvimento do Ensino, Ciência e Tecnologia do Estado de Mato Grosso do Sul (FUNDECT) (TO, 0054/13 and 025/2017), and Conselho Nacional de Desenvolvimento Científico e Tecnológico (CNPq). MBS was the recipient of a scholarship PIBIC-Fiocruz-CNPq - Program of scientific initiation.

\section{Availability of data and materials}

Data supporting the conclusions of this article are included within the article.

Ethics approval and consent to participate

Not applicable.

\section{Consent for publication}

Not applicable.

\section{Competing interests}

The authors declare that they have no competing interests.

\section{Author details}

${ }^{1}$ Laboratório de Biotecnologia, Instituto Nacional de Metrologia (INMETRO), Rio de Janeiro, RJ, Brazil. ${ }^{2}$ Universidade do Grande Rio -UNIGRANRIO, Rua Prof. José de Souza Herdy, 1.160 - Jardim 25 de Agosto, Duque de Caxias, RJ, Brazil. ${ }^{3}$ Laboratório de Ultraestrutura Celular Hertha Meyer (LUCHM, IBCCF-UFRJ), Universidade Federal do Rio de Janeiro, Ilha do Fundão, Rio de Janeiro, RJ, Brazil. ${ }^{4}$ Laboratório de Genômica Funcional e Bioinformática, Instituto Oswaldo Cruz (IOC, Fiocruz), Manguinhos, Rio de Janeiro, RJ, Brazil. ${ }^{5}$ Departamento de Patologia, Centro de Ciências Biológicas e da Saúde da UFMS, Universidade Federal do Mato Grosso do Sul, Cidade Universitária, Campo Grande, MS, Brazil. ${ }^{6}$ Fiocruz Mato Grosso do Sul, Rua Gabriel Abrão, 92 Jardim das Nações, Campo Grande, MS, Brazil. ${ }^{7}$ Laboratório de Biotecnologia e Fisiologia de Infecções Virais-LABIFIV, Instituto Oswaldo Cruz (IOC, Fiocruz), Av Brasil 4365, Manguinhos, Rio de Janeiro, RJ, Brazil.

Received: 30 July 2019 Accepted: 16 March 2020

Published online: 05 April 2020 


\section{References}

1. Granthon AC, Braga MV, Rodrigues JC, Cammerer S, Lorente SO, Gilbert $\mathrm{IH}$, et al. Alterations on the growth and ultrastructure of Leishmania chagasi induced by squalene synthase inhibitors. Vet Parasitol. 2007:146:25-34

2. de Souza W, Rodrigues JCF. Sterol biosynthesis pathway as target for antitrypanosomatid drugs. Interdiscip Perspect Infect Dis. 2009;2009:642502.

3. Fügi M, Gunasekera K, Ochsenreiter T, Guan X, Wenk MR, Mäser P. Genome profiling of sterol synthesis shows convergent evolution in parasites and guides chemotherapeutic attack. J Lipid Res. 2014;55:929-38.

4. WHO. Leishmaniasis in high-burden countries: an epidemiological update based on data reported in 2014. Wkly Epidemiol Rec 2016;91:285-96.

5. Ferreira GE, dos Santos BN, Dorval ME, Ramos TP, Porrozzi R, Peixoto AA, Cupolillo E. The genetic structure of Leishmania infantum populations in Brazil and its possible association with the transmission cycle of visceral leishmaniasis. PLoS ONE. 2012;7:e36242.

6. WHO. Control of the leishmaniases, vol. 949., World Health Organ Tech Rep SerGeneva: World Health Organization; 2010. p. 1-186.

7. Anversa L, Tiburcio MGS, Richini-Pereira VB, Ramirez LE. Human leishmaniasis in Brazil: a general review. Rev Assoc Med Bras. 2018;64:281-9.

8. Savioli L, Smith H, Thompson A. Giardia and Cryptosporidium join the neglected diseases initiative. Trends Parasitol. 2006;22:203-8.

9. Coelho $\mathrm{CH}$, Durigan M, Leal DAG, Schneider AB, Franco RMB, Singer SM. Giardiasis as a neglected disease in Brazil: systematic review of 20 years of publications. PLoS Negl Trop Dis. 2017;11:e0006005.

10. Heyworth MF. Giardia duodenalis genetic assemblages and hosts. Parasite. 2016;23:13.

11. Xiao L, Fayer R. Molecular characterisation of species and genotypes of Cryptosporidium and Giardia and assessment of zoonotic transmission. Int J Parasitol. 2008;38:1239-55.

12. Colli CM, Bezagio RC, Nishi L, Bignotto TS, Ferreira ÉC, Falavigna-Guilherme $\mathrm{AL}$, et al. Identical assemblage of Giardia duodenalis in humans, animals and vegetables in an urban area in southern Brazil indicates a relationship among them. PLOS ONE. 2015;10:e0118065.

13. Morrison HG, McArthur AG, Gillin FD, Aley SB, Adam RD, Olsen GJ, et al. Genomic minimalism in the early diverging intestinal parasite Giardia lamblia. Science. 2007;317:1921-6.

14. Voleman L, Najdrová V, Ástvaldsson Á, Tůmová P, Einarsson E, Švindrych Z, et al. Giardia intestinalis mitosomes undergo synchronized fission but not fusion and are constitutively associated with the endoplasmic reticulum. BMC Biol. 2017:15:27.

15. Ellis JE, Setchell KD, Kaneshiro ES. Detection of ubiquinone in parasitic and free-living protozoa, including species devoid of mitochondria. Mol Biochem Parasitol. 1994;65:213-24.

16. Chojnacki T, Dallner G. The biological role of dolichol. Biochem J. 1988;251:1-9.

17. Arruda DC, D'Alexandri FL, Katzin AM, Uliana SRB. Leishmania amazonensis: biosynthesis of polyprenols of 9 isoprene units by amastigotes. Exp Parasitol. 2008;118:624-8.

18. Grabińska KA, Cui J, Chatterjee A, Guan Z, Raetz CR, Robbins PW, Samuelson J. Molecular characterization of the cis-prenyltransferase of Giardia duodenalis. Glycobiology. 2010;20:824-32.

19. Srivastava A, Mukherjee P, Desai PV, Avery MA, Tekwani B. Structural analysis of farnesyl pyrophosphate synthase from parasitic protozoa, a potential chemotherapeutic target. Infect Disord Drug Targets. 2008;8:16-30.

20. Aripirala S, Gonzalez-Pacanowska D, Oldfield E, Kaiser M, Amzel LM, Gabelli SB. Structural and thermodynamic basis of the inhibition of Leishmania major farnesyl diphosphate synthase by nitrogen-containing bisphosphonates. Acta Crystallogr D Biol Crystallogr. 2014;70:802-10.

21. Ortiz-Gómez A, Jiménez C, Estévez AM, Juana Carrero-Lérida J, Ruiz-Pérez LM, Dolores González-Pacanowska D. Farnesyl diphosphate synthase is a cytosolic enzyme in Leishmania major promastigotes and its overexpression confers resistance to risedronate. Eukaryot Cell. 2006;5:1057-64.

22. Montalvetti A, Bailey BN, Martin MB, Severin GW, Oldfield E, Docampo R. Bisphosphonates are potent inhibitors of Trypanosoma cruzi farnesyl pyrophosphate synthase. J Biol Chem. 2001;276:33930-7.

23. Luckman SP, Hughes DE, Coxon FP, Graham R, Russell G, Rogers MJ. Nitrogen-containing bisphosphonates inhibit the mevalonate pathway and prevent post-translational prenylation of GTP-binding proteins, including Ras. J Bone Miner Res. 1998;13:581-9.
24. Coxon FP, Helfrich MH, Larijani B, Muzylak M, Dunford JE, Marshall D, et al. Identification of a novel phosphonocarboxylate inhibitor of Rab geranylgeranyl transferase that specifically prevents Rab prenylation in osteoclasts and macrophages. J Biol Chem. 2001;276:48213-22.

25. Garzoni LR, Caldera A, Meirelles MN, de Castro SL, Docampo R, Meints $\mathrm{GA}$, et al. Selective in vitro effects of the farnesyl pyrophosphate synthase inhibitor risedronate on Trypanosoma cruzi. Int J Antimicrob Agents. 2004;23:273-85.

26. Keister DB. Axenic culture of Giardia duodenalis in TYI-S-33 medium supplemented with bile. Trans R Soc Trop Med Hyg. 1983;77:487-8.

27. Madeira F, Park YM, Lee J, Buso N, Gur T, Madhusoodanan N, et al. The EMBL-EBI search and sequence analysis tools APIs in 2019. Nucleic Acids Res. 2019;47:W636-41.

28. Saitou N, Nei M. The neighbor-joining method: a new method for reconstructing phylogenetic trees. Mol Biol Evol. 1987:4:406-25.

29. Kumar S, Stecher G, Tamura K. MEGA7: molecular evolutionary genetics analysis version 7.0 for bigger datasets. Mol Biol Evol. 2016;33:1870-4.

30. Jones DT, Taylor WR, Thornton JM. The rapid generation of mutation data matrices from protein sequences. Comput Appl Biosci. 1992;8:275-82.

31. Felsenstein J. Confidence limits on phylogenies: an approach using the bootstrap. Evolution. 1985;39:783-91.

32. Webb B, Sali A. Protein structure modeling with MODELLER. Methods Mo Biol. 2014;1137:1-15.

33. Schrodinger LLC. The PyMOL molecular graphics system, version 1.8. Camberley: LLC; 2015

34. Henriques C, Moreira TLB, Maia-Brigagão C, Henriques-Pons A, Carvalho TMU, de Souza W. Tetrazolium salt-based methods for high-throughput evaluation of anti-parasite chemotherapy. Anal Methods. 2011;3:2148-55.

35. Fischer MJ, Meyer S, Claudel P, Bergdoll M, Karst F. Identification of a lysine residue important for the catalytic activity of yeast farnesyl diphosphate synthase. Protein J. 2011:30:334-9.

36. Picard MĖ, Nisole A, Béliveau C, Sen S, Barbar A, Shi R, Cusson M. Structural characterization of a lepidopteran type-II farnesyl diphosphate synthase from the spruce budworm, Choristoneura fumiferana: implications for inhibitor design. Insect Biochem Mol. Biol. 2017;92:84-92.

37. Hernandez PC, Wasserman M. Do genes from the cholesterol synthesis pathway exist and express in Giardia intestinalis? Parasitol Res. 2006;98:194-9.

38. Lee N, Bertholet S, Debrabant A, Muller J, Duncan R, Nakhasi HL. Programmed cell death in the unicellular protozoan parasite Leishmania. Cell Death Differ. 2002;9:53-64.

39. Park J, Lin YS, Tsantrizos YS, Berghuis AM. Structure of human farnesyl pyrophosphate synthase in complex with an aminopyridine bisphosphonate and two molecules of inorganic phosphate. Acta Crystallogr F Struct Biol Commun. 2014;70:299-304.

40. Ohnuma SI, Hirooka K, Ohto C, Nishino T. Conversion from archaeal geranylgeranyl diphosphate synthase to farnesyl diphosphate synthase. Two amino acids before the first aspartate-rich motif solely determine eukaryotic farnesyl diphosphate synthase activity. Biol Chem. 1996;272:5192-8.

41. Dhar MK, Koul A, Kaul S. Farnesyl pyrophosphate synthase: a key enzyme in isoprenoid biosynthetic pathway and potential molecular target for drug development. N Biotechnol. 2013;30:114-23.

42. Martin MB, Grimley JS, Lewis JC, Heath HT 3rd, Bailey BN, Kendrick H, et al. Bisphosphonates inhibit the growth of Trypanosoma brucei, Trypanosoma cruzi, Leishmania donovani, Toxoplasma gondii, and Plasmodium falciparum: a potential route to chemotherapy. J Med Chem. 2001;44:909-16.

43. Kessler RL, Soares MJ, Probst CM, Krieger MA. Trypanosoma cruzi response to sterol biosynthesis inhibitors: morphophysiological alterations leading to cell death. PLoS ONE. 2013;8:e55497.

44. Itzstein C, Coxon FP, Rogers MJ. The regulation of osteoclast function and bone resorption by small GTPases. Small GTPases. 2011;2:117-30.

45. Suri S, Mönkkönen J, Taskinen M, Pesonen J, Blank MA, Phipps RJ, Rogers MJ. Nitrogen-containing bisphosphonates induce apoptosis of Caco-2 cells in vitro by inhibiting the mevalonate pathway: a model of bisphosphonate-induced gastrointestinal toxicity. Bone. 2001;29:336-43.

46. Ali N, Jurczyluk J, Shay G, Tnimov Z, Alexandrov K, Munoz MA, et al. A highly sensitive prenylation assay reveals in vivo effects of bisphosphonate drug on the Rab prenylome of macrophages outside the skeleton. Small GTPases. 2015;6:202-11. 
47. Ghosh S, Chan JMW, Lea CR, Meints GA, Lewis JC, Tovian ZS, et al. Effects of bisphosphonates on the growth of Entamoeba histolytica and Plasmodium species in vitro and in vivo. J Med Chem. 2004;47:175-87.

48. Nuttall JM, Hettema EH, Watts DJ. Farnesyl diphosphate synthase, the target for nitrogen-containing bisphosphonate drugs, is a peroxisomal enzyme in the model system Dictyostelium discoideum. Biochem J. 2012:447:353-61.

49. Luján HD, Mowatt MR, Chen GZ, Nash TE. Isoprenylation of proteins in the protozoan Giardia lamblia. Mol Biochem Parasitol. 1995;72:121-7.

50. Anderson MS, Yarger JG, Burck CL, Poulter CD. Farnesyl diphosphate synthetase. Molecular cloning, sequence, and expression of an essential gene from Saccharomyces cerevisiae. J Biol Chem. 1989;264:19176-84.

51. Gabelli SB, McLellan JS, Montalvetti A, Oldfield E, Docampo R, Amzel LM Structure and mechanism of the farnesyl diphosphate synthase from Trypanosoma cruzi. Implications for drug design. Proteins. 2005;62:80-8.
52. Mao J, Mukherjee S, Zhang Y, Cao R, Sanders JM, Song Y, et al. T. brucei farnesyl diphosphate synthase complexed with minodronate. J Am Chem Soc. 2006;128:14485-97.

53. Hosfield DJ, Zhang Y, Dougan DR, Brooun A, Tari LW, Swanson RV, Finn J. Structural basis for bisphosphonate-mediated inhibition of isoprenoid biosynthesis. J Biol Chem. 2004;279:8526-9.

54. Cunillera N, Arró M, Delourme D, Karst F, Boronat A, Ferrer A. Arabidopsis thaliana contains two differentially expressed farnesyl-diphosphate synthase genes. J Biol Chem. 1996;271:7774-80.

\section{Publisher's Note}

Springer Nature remains neutral with regard to jurisdictional claims in published maps and institutional affiliations.
Ready to submit your research? Choose BMC and benefit from:

- fast, convenient online submission

- thorough peer review by experienced researchers in your field

- rapid publication on acceptance

- support for research data, including large and complex data types

- gold Open Access which fosters wider collaboration and increased citations

- maximum visibility for your research: over $100 \mathrm{M}$ website views per year

At BMC, research is always in progress.

Learn more biomedcentral.com/submissions 